\title{
Preservation of Classes of Discrete Distributions Under Reliability Operations
}

\author{
I. Elbatal ${ }^{1}$ and M. Ahsanullah ${ }^{2}$ \\ ${ }^{1}$ Institute of Statistical Studies and Research, Department of Mathematical Statistics, Cairo University \\ ${ }^{2}$ Department of Management Sciences, Rider University, Lawrenceville, New Jersey, USA \\ i_elbatal@staff.cu.edu.eg, ahsan@rider.edu
}

Received 5 January 2012

Accepted 6 June 2012

\begin{abstract}
In this paper we consider some widely utilized classes of discrete distributions and aim to provide a systematic overview about their preservation under convolution. This paper will serve as a detailed reference for the study and applications of the preservation of the discrete NBU(2), NBUCA classes of discrete distributions.
\end{abstract}

Keywords: discrete ageing classes; NBUC, NBUCA; NBU(2), Convolution.

\section{Introduction and Motivation}

The literature on reliability theory mainly deals with non-negative absolutely continuous random variables. However, quiet often we come across with situations where the product life can be described through non-negative integer valued random variable. For example (i) a device can be monitored only once per time period and the observation is taken as the number of time periods successfully completed prior to the failure of a device, (ii) a piece of equipment may operate in cycles and we measure the number of cycles completed prior to failure, for example number of copies made by a photocopier before it fails, (iii) number of road accidents in a city in a given month. See Chen and Manatunga (2007), Kemp (2004) and Yu (2007) and the references therein. Therefore we need to develop tools, analogous to the continuous case, for studying the discrete failure data.

Discrete lifetimes usually arise through grouping or finite-precision measurement of continuous time phenomena. They may also be found naturally where failure may occur only at the instants of shock. Parametric models for discrete life distributions may be found in Bain (1991), Adams, Watson (1989) and Xekalaki (1983). Nonparametric families of discrete life distributions has been considered in the reliability literature mainly in connection with shock models leading to various continuous-time ageing families See Barlow and Proschan (1982), Roy and Gupta (1992), among others. have studied definitions, interrelations and closure properties of some nonparametric ageing families of distributions having a finite support. 
Let $X$ be a non-negative discrete random variable representing the lifetime of the unit. Without loss of generality we will assume the $\mathbb{N}_{+}$is support of $X$. The probability mass function (p.m.f.) given by $f(x)=\operatorname{Pr}\{X=x\}, x=0,1, \ldots$, the cumulative distribution function $F$ of $X$ satisfies $F(x)=\operatorname{Pr}\{X \leqslant x\}=\sum_{i=0}^{x} f(i)$ for all $x \in \mathbb{N}$, and the survival function $\bar{F}$ of $X$ satisfies $\bar{F}(x)=$ $1-F(x)=\sum_{i=x+1}^{\infty} f(i)$ for all $x \in \mathbb{N}$ where $\mathbb{N}=\{0,1, \ldots\}$. Furthermore, for all $x=-1,-2, \ldots$, we have $f(x)=0, F(x)=0$, and $\bar{F}(0)=1$. The distribution of a counting random variable is called a discrete life distribution. In particular, if $f(0)=\operatorname{Pr}\{X=0\}=0$, or a counting random variable $X$ has a support on $\mathbb{N}_{+}=\{1,2, \ldots\}$, we say that the discrete distribution is zero-truncated. Moreover, we denote $\mathbb{N}_{-}=\{-1,0,1, \ldots\}$.

The following concepts plays a fundamental role in reliability theory, life testing, inventory theory, and Biometry. It is one of the most widely used mathematical ways of describing ageing. Recently, Pavlova et al. (2006) defined discrete hazard rate of $F$ by the following

$$
h_{F}(x)=\frac{f(x)}{f(x)+\bar{F}(x)}, \text { for } x \in \mathbb{N} \text {, and } P\{X \geqslant x\}>0 .
$$

Alternative names for the function $h_{F}(x)$ are failure rate, force of mortality, etc. The discrete mean residual lifetime of $F$ is defined by

$$
M_{F}(x)=E(X-x \mid X>x)=\frac{\sum_{i=x}^{\infty} \bar{F}(i)}{\bar{F}(x)}, \text { for } x \in \mathbb{N} \text {, and } \bar{F}(x)>0 .
$$

Similar to continuous distributions, discrete distributions can be also classified by the properties of the failure rates, mean residual lifetimes, and survival functions of discrete distributions. Some commonly used classes of discrete distributions include the classes of discrete decreasing failure rate $(d-D F R)$, discrete decreasing failure rate average $(d-D F R A)$, discrete new worse than used $(d$ $N W U)$, discrete increasing mean residual lifetime ( $d$-IMRL), discrete harmonic new worse than used in expectation $(d-H N W U E)$, and their dual ones including the classes of discrete increasing failure rate $(d-I F R)$, discrete increasing failure rate average $(d-I F R A)$, discrete new better than used $(d$ $N B U)$, discrete decreasing mean residual lifetime ( $d-D M R L)$, and discrete harmonic new better than used in expectation $(d-H N B U E)$.

These classes of discrete distributions have been used extensively in different fields of statistics and probability such as insurance, finance, reliability, survival analysis, and others. See, for example, Barlow and Proschan (1975), Cai and Kalashnikov (2000), Cai and Willmot (2005), Fagiuoli and Pellerey (1994), Gupta et al. (1997), Hu et al. (2003), Ross (1996), Shaked and Shanthikumar (1994), Shaked et al. (1995), Willmot and Cai (2001), Willmot and Lin (2000) and references therein.

Ahmad et al. (2006) defined the class of new better than used in increasing convex average order $(N B U C A)$. This class is requiring the distribution function $F$ of a random variable $X$ to satisfy

$$
\int_{0}^{\infty} \int_{x}^{\infty} \bar{F}(u+t) d u d t \leqslant \bar{F}(t) \int_{0}^{\infty} \int_{x}^{\infty} \bar{F}(u) d u d x \text {, for all } t \geqslant 0 .
$$

Also, $\mathrm{Li}$ and Kochar (2001) define a continuous random variable $X$ (or its distribution function $F$ ) to be new better than used of second order $(N B U(2))$ if

$$
\int_{0}^{x} \bar{F}(t+y) d y \leqslant \bar{F}(t) \int_{0}^{x} \int_{x}^{\infty} \epsilon_{x}^{\infty} \bar{F}(y) d y, \text { for all } x, t \geqslant 0 .
$$


Both authors of the two classes have demonstrated their usefulness in reliability applications as well in as other fields.

This paper is organized as follows: In Section 2, we defined the analogous discrete distributions and we give the definitions of some classes of discrete distributions. The properties of the discrete new better than used in the convex and second order discussed in Section 3. Finally, in Section 4, we establish the convolution preservation of the discrete new better than used in convex average order class.

\section{Basic Definitions}

Most of the nonparametric discrete classes of distributions that are commonly found in the reliability literature are based on some notion of aging. In this section we present the definitions of some classes of discrete distributions, which will be used in the sequel.

Definition 2.1. Let $X$ and $Y$ two non-negative random variables with distribution functions $F(x)$ and $G(x)$, and survival functions $\bar{F}(x)$ and $\bar{G}(x)$ respectively. $X$ is said to be smaller than $Y$ in the (i) usual stochastic order, denoted by $X \leqslant_{s t} Y$, if

$$
\bar{F}(x) \leqslant \bar{G}(x) \text {, for all } x .
$$

(ii) Discrete increasing convex order, denoted by $X \leqslant_{d i c x} Y$, if

$$
\sum_{i=x}^{\infty} \bar{F}(i) \leqslant \sum_{i=x}^{\infty} \bar{G}(i), \text { for all } i \in \mathbb{N} .
$$

(iii) Discrete increasing convex average order, denoted by $X \leqslant_{\text {dicxa }} Y$, if

$$
\sum_{x=0}^{\infty} \sum_{i=x}^{\infty} \bar{F}(i) \leqslant \sum_{x=0}^{\infty} \sum_{i=x}^{\infty} \bar{G}(i) \text {, for all } i \in \mathbb{N} .
$$

(iv) Discrete increasing concave order, denoted by $X \leqslant_{\text {dicv }} Y$, if

$$
\sum_{i=0}^{x} \bar{F}(i) \geqslant \sum_{i=0}^{x} \bar{G}(i), \text { for all } i \in \mathbb{N} .
$$

Definition 2.2. A discrete distribution $F$ is called discrete new better than used in convex ordering $(d-N B U C)$ (or discrete worse better than used in convex ordering $(d-N W U C)$ iff

$$
\sum_{i=x}^{\infty} \bar{F}(i+t) \leqslant(\geqslant) \bar{F}(t) \sum_{i=x}^{\infty} \bar{F}(i), \text { for all } x, t \in \mathbb{N} .
$$

Definition 2.3. An integer-valued random variable $X$ (or its cdf $F$ ) is said to be discrete new better than used in convex average order (denoted by $d-N B U C A$ ) if

$$
\sum_{y=0}^{\infty} \sum_{i=0}^{\infty} \bar{F}(x+y+i) \leqslant \sum_{y=0}^{\infty} \bar{F}(y) \sum_{i=0}^{\infty} \bar{F}(x+i) .
$$

Definition 2.4. An integer-valued random variable $X$ (or its $\operatorname{cdf} F$ ) is said to be discrete new better than used in second order (denoted by $d-N B U(2))$ if

$$
\sum_{j=0}^{k} \bar{F}(i+j) \leqslant \bar{F}(i) \sum_{j=0}^{k} \bar{F}(j), \text { for all } i, j \in \mathbb{N} .
$$


Definition 2.5. A discrete distribution $F$ with finite mean $\mu$ is said to be discrete new better than used in expectation $(d-N B U E)$ if for all $x \in \mathbb{N}$.

$$
\sum_{i=x}^{\infty} \bar{F}(i) \leqslant \bar{F}(x) \sum_{j=0}^{\infty} \bar{F}(j), \text { for all } x \in \mathbb{N} .
$$

or equivalently,

$$
\sum_{i=x}^{\infty} \bar{F}(i) \leqslant \mu \bar{F}(x)
$$

Definition 2.6. A discrete distribution $F$ with expectation $\sum_{i=0}^{\infty} \bar{F}(i)=\mu$ is called discrete harmonic new better than used in expectation $(d-H N B U E)$ if for all $x \in \mathbb{N}$.

$$
\sum_{i=x}^{\infty} \bar{F}(i) \leqslant \mu\left(1-\frac{1}{\mu}\right)^{x} .
$$

The following theorem state the relation between discrete new better than used in second order and discrete new better than used in expectation.

Theorem 2.1. If $X \in d-N B U(2)$, then $X \in d-N B U E$.

Proof. Since $X$ is $d-N B U(2)$, then

$$
\sum_{j=0}^{k} \bar{F}(i+j) \leqslant \bar{F}(i) \sum_{j=0}^{k} \bar{F}(j), \text { for all } k \in \mathbb{N} .
$$

Letting $k$ tends to infinity in equation (2.3), on gets

$$
\sum_{j=0}^{\infty} \bar{F}(i+j) \leqslant \bar{F}(i) \sum_{j=0}^{\infty} \bar{F}(j)
$$

or equivalently

$$
\sum_{j=0}^{\infty} \bar{F}(i+j) \leqslant \bar{F}(i) \mu
$$

which is $d$-NBUE.

\section{The Properties of $d-N B U C$ and d-NBU(2) Classes}

In this section we presented characterizations for the discrete new better than used in convex order and the discrete new better than used in the second order. This section contained two subsections. In the first one, we proved that $d-N B U C$ class is closed under formation of parallel system. In the second one, we demonstrate preservation of $d-N B U(2)$ under convolution.

\subsection{Closure of d-NBUC under formation of parallel system}

In this subsection we prove that the discrete new better than used in convex order class is closed under formation of parallel systems with independent and identically distributed components.

Theorem 3.1. Let $X_{1}, X_{2}, \ldots, X_{n}$ be independent and identically distributed integer-valued random variables with distribution function $F$. Let $X_{(n)}=\operatorname{Max}_{1 \leqslant i \leqslant n} X_{i}$. Then $X_{(n)}$ has d-NBUC property. 
Proof. The survival function of $X_{(n)}$ is

$$
\bar{F}_{(n)}(t)=P\left(X_{(n)}>t\right)=1-F^{n}(t)
$$

we need show that

$$
\sum_{i=x}^{\infty} \bar{F}_{(n)}(i+t) \leqslant \bar{F}_{(n)}(t) \sum_{i=x}^{\infty} \bar{F}_{(n)}(i)
$$

the inequality (3.2) obviously holds whenever $\bar{F}_{(n)}(t)=0$, and for $\bar{F}_{(n)}(t) \neq 0$, the characterization result given by (2.1) may be reformulated as $F \in \mathrm{d}$-NBUC iff

$$
\frac{F(t)}{1-F(t)} \sum_{j=t+x}^{\infty} \bar{F}(j) \leqslant \sum_{j=x}^{x+t}(1-F(j))
$$

the right hand side of (3.3) satisfies the following inequality

$$
\sum_{j=x}^{x+t} \bar{F}(j) \leqslant \sum_{j=x}^{x+t} \bar{F}_{(n)}(j)
$$

and the left hand side of (3.4) satisfies

$$
\frac{F(t)}{1-F(t)} \sum_{j=t+x}^{\infty} \bar{F}(j) \geqslant \sum_{j=t+x}^{\infty} \frac{F^{n}(t)\left(1-F^{n}(j)\right)}{1-F^{n}(t)}
$$

the last inequality (3.5) holds because

$$
\begin{aligned}
& \sum_{j=t+x}^{\infty}\left\{\frac{F(t)(1-F(j))}{1-F(t)}-\frac{F^{n}(t)\left(1-F^{n}(j)\right)}{1-F^{n}(t)}\right\} \\
= & \sum_{j=t+x}^{\infty} \frac{F(t)(1-F(j))}{1-F(t)}\left\{1-F^{n-1}(t)\left[\frac{[1-F(t)]\left[1-F^{n}(j)\right]}{\left[1-F^{n}(t)[1-F(j)]\right]}\right]\right\} \\
= & \sum_{j=t+x}^{\infty} \frac{F(t)(1-F(j))}{1-F(t)}\left\{1-F^{n-1}(t)\left[\frac{1+F(j)+\cdots+F^{n-1}(j)}{1+F(t)+\cdots+F^{n-1}(t)}\right]\right\} \\
\geqslant & \sum_{j=t+x}^{\infty} \frac{F(t)(1-F(j))}{1-F(t)}\left\{1-\frac{F^{n-1}(t)\left[1+F^{-1}(t)+\cdots+F^{-(n-1)}(t)\right]}{\left[1+F(t)+\cdots+F^{n-1}(t)\right]}\right\} \\
\geqslant & 0 .
\end{aligned}
$$

Since

$$
F(j) \leqslant F^{-1}(j) \leqslant F^{-1}(t), \text { for all } j \geqslant t
$$

we get

$$
\sum_{j=t+x}^{\infty} \frac{F(t)(1-F(j))}{1-F(t)} \geqslant \sum_{j=t+x}^{\infty} \frac{F_{(n)}(t)}{\bar{F}_{(n)}(t)} \bar{F}_{(n)}(j)
$$

from (3.5) and (3.6) we get

$$
\sum_{j=t+x}^{\infty} \frac{F_{(n)}(t)}{\bar{F}_{(n)}(t)} \bar{F}_{(n)}(j) \leqslant \sum_{j=x}^{x+t} \bar{F}_{(n)}(j)
$$


or

$$
\sum_{j=t+x}^{\infty}\left[\frac{1}{\bar{F}_{(n)}(t)}-1\right] \bar{F}_{(n)}(j) \leqslant \sum_{j=x}^{x+t} \bar{F}_{(n)}(j)
$$

or

$$
\sum_{j=t+x}^{\infty} \frac{\bar{F}_{(n)}(j)}{\bar{F}_{(n)}(t)} \leqslant \sum_{j=x}^{\infty} \bar{F}_{(n)}(j)
$$

this means that

$$
\sum_{j=t+x}^{\infty} \bar{F}_{(n)}(j) \leqslant \bar{F}_{(n)}(t) \sum_{j=x}^{\infty} \bar{F}_{(n)}(j) .
$$

Thus

$$
\sum_{i=x}^{\infty} \bar{F}_{(n)}(i+t) \leqslant \bar{F}_{(n)}(t) \sum_{i=x}^{\infty} \bar{F}_{(n)}(i)
$$

This proves the required result.

\subsection{Preservation of $d-N B U(2)$ class under convolution}

In this subsection we discuss preservation of $d-N B U(2)$ under convolution.

Theorem 3.2. Suppose that $F_{1}$ and $F_{2}$ are two independent $d-N B U(2)$ life distributions. Then their convolution is also d-NBU(2).

\section{Proof.}

$$
\begin{aligned}
\sum_{s=0}^{x} \bar{F}(t+s) & =\sum_{k=0}^{x} \sum_{s=0}^{\infty} \bar{F}_{1}(t+s-k) f_{2}(k) \\
& =\left\{\sum_{s=0}^{x} \sum_{k=0}^{t} \bar{F}_{1}(t+s-k) f_{2}(k) \sum_{s=0}^{x} \sum_{k=t+1}^{\infty} \bar{F}_{1}(t+s-k) f_{2}(k)\right\} \\
& =\left\{\sum_{k=0}^{t}\left[\sum_{s=0}^{x} \bar{F}_{1}(t+s-k)\right] f_{2}(k)+\sum_{s=0}^{x}\left[\sum_{v=0}^{\infty} \bar{F}_{1}(s-v) F_{2}(v+t)\right]\right\} \\
& =A_{1}+A_{2}
\end{aligned}
$$

where

$$
A_{1} \leqslant \sum_{k=0}^{t}\left[\bar{F}_{1}(t-k) \sum_{s=0}^{x} \bar{F}_{1}(s) f_{2}(k)\right]
$$


and

$$
\begin{aligned}
A_{2} & \leqslant \sum_{s=0}^{x}\left[\bar{F}_{1}(s) \bar{F}_{2}(t)+\sum_{s=0}^{\infty} \sum_{v=0}^{\infty} \bar{F}_{2}(v+t) f_{1}(s-v)\right] \\
& =\bar{F}_{2}(t) \sum_{k=0}^{x} \bar{F}_{1}(k)+\sum_{s=0}^{x}\left[\sum_{v=0}^{s} \bar{F}_{2}(v+t) f_{1}(s-v)\right] \\
& =\Psi+\sum_{s=0}^{x}\left[\sum_{k=0}^{s} \bar{F}_{2}(t+s-k) f_{1}(k)\right] \\
& =\Psi+\sum_{k=0}^{x}\left[\sum_{s=k}^{x} \bar{F}_{2}(t+s-k)\right] f_{1}(k) \\
& =\Psi+\sum_{k=0}^{x}\left[\sum_{s=0}^{x-k} \bar{F}_{2}(t+s)\right] f_{1}(k) \\
& \leqslant \Psi+\bar{F}_{2}(t) \sum_{k=0}^{x} \sum_{s=0}^{x-k} \bar{F}_{2}(s) f_{1}(k) \\
& =\Psi+\bar{F}_{2}(t) \sum_{k=0}^{x} \sum_{k=0}^{s} \bar{F}_{2}(s-k) f_{1}(k) .
\end{aligned}
$$

where the inequality follows from the $d-N B U(2)$ property of $F_{2}$ and

$$
\Psi=\bar{F}_{2}(t) \sum_{k=0}^{x} \bar{F}_{1}(k)
$$

On the other hand

$$
\begin{aligned}
\bar{F}(t) \sum_{s=0}^{x} \bar{F}(s) & =\bar{F}(t) \sum_{k=0}^{x} \sum_{s=0}^{\infty} \bar{F}_{2}(s-k) f_{1}(k) \\
& =\bar{F}(t)\left[\sum_{k=0}^{x} \bar{F}_{1}(k)+\sum_{s=0}^{x} \sum_{k=0}^{s} \bar{F}_{2}(s-k) f_{1}(k)\right] \\
& \geqslant A_{1}+A_{2}=\sum_{s=0}^{x} \bar{F}(t+s) .
\end{aligned}
$$

from (3.7), (3.8) and (3.9) and the fact that $\bar{F}(t) \geqslant \bar{F}_{2}(t)$ for all $t \geqslant 0$. This proves that $F$ is $d$ $\operatorname{NBU}(2)$.

\section{Discrete NBUCA Class Preservation Under Convolution}

As an important reliability operation, convolution of life distributions of certain class is often paid much attention. It has been shown that both the $d-N B U$ and $d-N B U C$ classes are closed under this operation, see Pavlova et al. (2006). In this section we established the closure property of the $d$ NBUCA class under the convolution operation.

Theorem 4.1. Suppose that $F_{1}$ and $F_{2}$ are two independent $d$-NBUCA life distributions. Then their convolution is also d-NBUCA. 


\section{Elbatal and M. Ahsanullah}

Proof. The survival function of convolution of two life distribution $F_{1}$ and $F_{2}$ is

$$
\bar{F}(u)=\sum_{t=0}^{u} \bar{F}_{1}(u-t) f_{2}(t), \text { for all } u \in \mathbb{N} .
$$

Let $x, y \in \mathbb{N}$. On one hand

$$
\begin{aligned}
\sum_{y=0}^{\infty} \sum_{i=0}^{\infty} \bar{F}(x+y+i) & =\sum_{y=0}^{\infty} \sum_{i=0}^{\infty} \sum_{j=0}^{\infty} \bar{F}_{1}(x+y+i-j) f_{2}(j) \\
& =\left\{\sum_{y=0}^{\infty} \sum_{i=0}^{\infty} \sum_{j=0}^{x-1} \bar{F}_{1}(x+y+i-j) f_{2}(j)+\sum_{y=0}^{\infty} \sum_{i=0}^{\infty} \sum_{j=x}^{\infty} \bar{F}_{1}(x+y+i-j) f_{2}(j)\right\} \\
& =\left\{\sum_{y=0}^{\infty} \sum_{j=0}^{x-1} f_{2}(j) \sum_{i=0}^{\infty} \bar{F}_{1}(x+y+i-j)+\sum_{y=0}^{\infty} \sum_{i=0}^{\infty} \sum_{j=0}^{\infty} \bar{F}_{1}(y+i-j) f_{2}(x+j)\right\} \\
& =I_{1}+I_{2}
\end{aligned}
$$

Observe that

$$
\begin{aligned}
I_{1} & \leqslant \sum_{y=0}^{\infty} \bar{F}_{1}(y) \sum_{j=0}^{x-1} f_{2}(j) \sum_{i=0}^{\infty} \bar{F}_{1}(x+i-j) \\
& \leqslant \sum_{y=0}^{\infty} \bar{F}(y) \sum_{i=0}^{\infty} \sum_{j=0}^{x-1} \bar{F}_{1}(x+i-j) f_{2}(j) .
\end{aligned}
$$

and

$$
\begin{gathered}
I_{2}=\sum_{y=0}^{\infty} \sum_{i=0}^{\infty} \bar{F}_{2}(x-1) \sum_{i=y}^{\infty} \bar{F}_{1}(i)+\sum_{y=0}^{\infty} \sum_{i=0}^{\infty} \sum_{j=0}^{\infty} \bar{F}_{2}(x+j) f_{1}(y+i-j) \\
=\sum_{y=0}^{\infty} \sum_{i=y}^{\infty} \bar{F}_{2}(x-1) \bar{F}_{1}(i)+\sum_{y=0}^{\infty} \sum_{i=0}^{\infty} \sum_{j=0}^{y+i} \bar{F}_{2}(x+y+i-j) f_{1}(j) \\
=\bar{F}_{2}(x-1) \sum_{y=0}^{\infty} \sum_{i=y}^{\infty} \bar{F}_{1}(i)+\sum_{y=0}^{\infty} \sum_{j=y+1}^{\infty} \sum_{i=j-y}^{\infty} \bar{F}_{2}(x+y+i-j) f_{1}(j) \\
\quad+\sum_{y=0}^{\infty} \sum_{i=0}^{\infty} \sum_{j=0}^{\infty} \bar{F}_{2}(x+y+i-j) f_{1}(j) \\
=A_{1}+A_{2}+A_{3},
\end{gathered}
$$


where

$$
\begin{aligned}
A_{1} & \leqslant \sum_{y=0}^{\infty} \bar{F}_{2}(x-1) \mu_{1} \bar{F}_{1}(y) \\
& \leqslant \mu_{1} \mu \bar{F}_{2}(x-1), \\
A_{2}= & \sum_{y=0}^{\infty} \sum_{j=y+1}^{\infty} f_{1}(j) \sum_{i=0}^{\infty} \bar{F}_{2}(x+i) \\
= & \sum_{y=0}^{\infty} \bar{F}_{1}(y) \sum_{i=0}^{\infty} \bar{F}_{2}(x+i), \\
A_{3} \leqslant & \sum_{y=0}^{\infty} I \sum_{j=0}^{y} f_{1}(j)\left[\bar{F}_{2}(y-j) \sum_{i=0}^{\infty} \bar{F}_{2}(x+i)\right] \\
= & \sum_{y=0}^{\infty}\left[\sum_{j=0}^{y} \bar{F}_{2}(y-j) f_{1}(j)\right] \sum_{i=0}^{\infty} \bar{F}_{2}(x+i) \\
= & \sum_{y=0}^{\infty}\left[\bar{F}(y)-\bar{F}_{1}(y)\right] \sum_{i=0}^{\infty} \bar{F}_{2}(x+i) \\
= & \left(\mu-\mu_{1}\right) \sum_{i=0}^{\infty} \bar{F}_{(x+i) .}
\end{aligned}
$$

Hence

$$
I_{2} \leqslant \mu_{1} \mu \bar{F}_{2}(x-1)+\mu \sum_{i=0}^{\infty} \bar{F}_{2}(x+i) .
$$

Combining Equations (4.1), (4.2) and (4.3) we see that

$$
\sum_{y=0}^{\infty} \sum_{i=0}^{\infty} \bar{F}(x+y+i) \leqslant \mu\left\{\left\{\sum_{i=0}^{\infty} \sum_{i=0}^{x-1} \bar{F}_{1}(x+i-j) f_{2}(j)+\mu_{1} \bar{F}_{2}(x-1)+\bar{F}_{2}(x+i)\right\}\right\} .
$$

Finally, implementing Equation (4.2) with $y=0$, we get

$$
\begin{gathered}
\sum_{y=0}^{\infty} \bar{F}(y) \sum_{i=0}^{\infty} \bar{F}(x+i)=\sum_{y=0}^{\infty} \bar{F}(y)\left\{\sum_{i=0}^{\infty} \sum_{i=0}^{x-1} \bar{F}_{1}(x+i-j) f_{2}(j)+\mu_{1} \bar{F}_{2}(x-1)\right. \\
\left.+\sum_{j=0}^{\infty} \sum_{i=j}^{\infty} \bar{F}_{2}(x+i-j) f_{1}(j)\right\} \\
=\sum_{y=0}^{\infty} \bar{F}(y)\left\{\sum_{i=0}^{\infty} \sum_{i=0}^{x-1} \bar{F}_{1}(x+i-j) f_{2}(j)+\mu_{1} \bar{F}_{2}(x-1)\right. \\
\left.+\sum_{j=0}^{\infty} f_{1}(j) \sum_{i=0}^{\infty} \bar{F}_{2}(x+i)\right\} \\
\geqslant \sum_{y=0}^{\infty} \sum_{i=0}^{\infty} \bar{F}(x+y+i) .
\end{gathered}
$$

which means that $F$ is $d-N B U C A$. 


\section{References}

[1] Adams G. and Watson R. (1989). A discrete time parametric model for the analysis of failure time data, Aust. J. Statistics 31, 365-384.

[2] A. Ahmad, H. Ahmed, I. Elbatal and M. Kayid. (2006). An Ageing Notion Derived From the Increasing Convex Ordering: the NBUCA class. Journal of Statistical Planing and Inference, USA, 136, 555-569.

[3] Bain L.J. (1991). Statistical Analysis of Reliability and Life Testing Models. Marcel Dekker, New York.

[4] Barlow, R.E. and Proschan, F. (1981). Statistical Theory of Reliability and Life Testing: Probability Models. Holt, Rinehart and Winston, Inc.

[5] Cai, J. and Kalashnikov, V. (2000). NWU property of a class of random sums. Journal of Applied Probability 37, 283-289.

[6] Cai, J. and Willmot, G. (2005). Monotonicity and aging properties of random sums. Statistics and Probability Letters, 73, 381-392.

[7] Chen, S. and Manatunga, A.K. (2007). A note on proportional hazards and proportional odds models, Statistics and Probability Letters, 77, 981-988.

[8] Ching, K. and Zongfu, H. (1989). Some criteria for aging. J. Amer. Assoc., 64, 1472-1483.

[9] Fagiuoli, E., and Pellerey, F. (1994). Preservation of certain classes of life distribution under Poisson shock models. Journal of Applied Probability, 31, 458-465.

[10] Gupta, P.L., Gupta, R.C. and Tripathi, R.C., (1997). On the monotonic properties of discrete failure rates. Journal of Statistics Planning and Inference, 65, 255-268.

[11] $\mathrm{Hu}, \mathrm{T} ., \mathrm{Ma}, \mathrm{M} .$, Nanda, A.K. (2003). Moment inequalities for discrete aging families. Communication in Statistics, Part A. Theory and Methods, 32, 61-91.

[12] Kemp, A.W. (2004). Classes of discrete life distributions, Communications in Statistics - Theory and Methods, 33, 3069-3093.

[13] Li, X.H., Kochar, S.C. (2001). Some new results involving the NBU(2) lass of life distributions. J. Appl. Probab., 38, 242-247.

[14] Pavlova, K., and Willmot, E. (2006). The preservation of classes of discrete distributions under convolution and mixing. Insurance Mathematics and Economics, 38, 391-405.

[15] Ross, S. (1996). Stochastic Processes, second ed. John Wiley, New York.

[16] Roy, D. and Gupta, R.P. (1992). Characterizations and model selections through reliability measures in the discrete case, Statistics and Probability Letters, 43, 197-206.

[17] Shaked, M. and Shanthikumar, G.J. (1994). Stochastic Orders and their Applications. Academic Press, New York.

[18] Shaked, M. Shanthikumar, G.J. and Valdez-Torres, J. (1995). Discrete hazard rate functions. Computers and Operation Research, 22, 391-402.

[19] Xekalaki, E. (1983). Hazard function and life distributions in discrete time, Communications in Statistics- Theory and Methods, 12, 2503-2509.

[20] Willmot, G. and Cai, J. (2001). Aging and other distributional properties of discrete compound geometric distributions. Insurance: Mathematics and Economics, 28, 361-379.

[21] Willmot, G.E. and Lin, X.S. (2000). Lundberg Approximations for Compound Distributions with Insurance Applications. Springer, New York.

[22] Xekalaki, E. (1983). Hazard function and life distributions in discrete time, Communications in Statistics - Theory and Methods, 12, 2503-2509.

[23] Yu, Q. (2007). A note on the proportional hazards model with discontinuous data, Statistics and Probability Letters, 77, 735-739. 